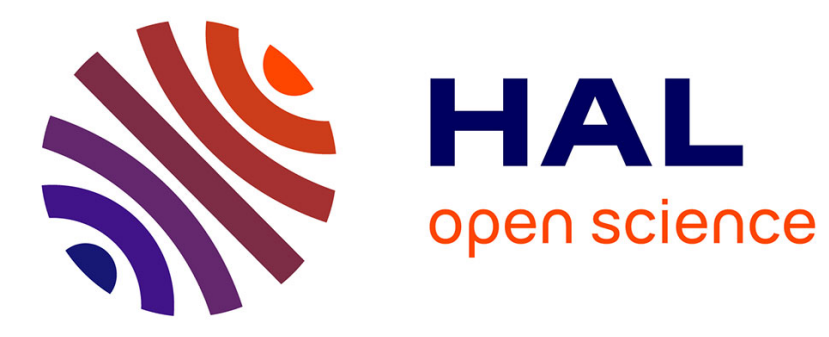

\title{
Where does the droplet size distribution come from?
}

Romain Canu, Stefano Puggelli, Mohammed Essadki, Benjamin Duret, Thibaut Menard, Marc Massot, Julien Reveillon, F.X. Demoulin

\section{To cite this version:}

Romain Canu, Stefano Puggelli, Mohammed Essadki, Benjamin Duret, Thibaut Menard, et al.. Where does the droplet size distribution come from?. International Journal of Multiphase Flow, 2018, 107, pp.230-245. 10.1016/j.ijmultiphaseflow.2018.06.010 . hal-01821941v2

\section{HAL Id: hal-01821941 \\ https://hal.science/hal-01821941v2}

Submitted on 30 Nov 2018

HAL is a multi-disciplinary open access archive for the deposit and dissemination of scientific research documents, whether they are published or not. The documents may come from teaching and research institutions in France or abroad, or from public or private research centers.
L'archive ouverte pluridisciplinaire HAL, est destinée au dépôt et à la diffusion de documents scientifiques de niveau recherche, publiés ou non, émanant des établissements d'enseignement et de recherche français ou étrangers, des laboratoires publics ou privés. 


\title{
Where does the droplet size distribution come from?
}

\author{
Romain Canu ${ }^{\mathrm{a}}$, Stefano Puggellid ${ }^{\mathrm{d}}$, Mohamed Essadki ${ }^{\mathrm{b}}$, Benjamin Duret ${ }^{\mathrm{a},{ }^{*} \text {, }}$ \\ Thibaut Menard $^{\mathrm{a}}$, Marc Massot ${ }^{\mathrm{c}}$, Julien Reveillon ${ }^{\mathrm{a}}$, F.X. Demoulin ${ }^{\mathrm{a}}$ \\ ${ }^{a}$ CNRS UMR 6614 - CORIA Normandie Université, Université et INSA de Rouen, Site \\ Universitaire du Madrillet, BP 12, 76801, Saint Etienne du Rouvray cedex, France \\ ${ }^{b}$ IFP Energies nouvelles, 1-4 Avenue du Bois Préau, 92852 Rueil-Malmaison, France \\ ${ }^{c}$ Centre de Mathématiques Appliquées, Ecole polytechnique, CNRS, Université \\ Paris-Saclay, Route de Saclay, 91128 Palaiseau Cedex, FRANCE \\ ${ }^{d}$ Department of Industrial Engineering, University of Florence, 50139, via S. Marta 3, \\ Florence, Italy
}

\begin{abstract}
This study employs DNS of two-phase flows to enhance primary atomization understanding and modeling to be used in numerical simulation in RANS or LES framework. In particular, the work has been aimed at improving the information on the liquid-gas interface evolution for modeling approaches, such as the Eulerian-Lagrangian Spray Atomization (ELSA) framework. Even though this approach has been already successfully employed to describe the complete liquid atomization process from the primary region to the dilute spray, improvements are still expected on the derivation of the drop size distribution (DSD). The main aim of the present work is the introduction of a new framework to achieve a continuous description of the DSD formation during the atomization process. The attention is here focused on the extraction from DNS data of the behavior of geometrical variable of the liquid-gas interface, such as the mean $(\mathrm{H})$ and Gauss (G) surface curvatures. The use of a Surface Curvature Distribution is also proposed and studied.
\end{abstract}

A Rayleigh-Plateau instability along a column of liquid and a droplet collision case are first of all considered to analyze and to verify the capabilities of the code to correctly predicting the curvature distributions. A statistical analysis

*Corresponding author : duret@coria.fr 
based on the curvatures data, in terms of probability density function, is presented in order to determine the physical parameters that control the curvatures on this test case. Then, the same formulation is applied in the analysis of the two phase Homogeneous Isotropic Turbulence (HIT) configuration to study how the curvatures evolve all along the atomization process. Joint PDFs are used to illustrate the topological changes of the interface when increasing the liquid volume fraction.

Keywords: interface, curvature, DNS, two-phase flows 


\begin{tabular}{|c|c|c|}
\hline symbol & definition & unit \\
\hline$D$ & Droplet diameter & $\mathrm{m}$ \\
\hline$G$ & Gauss curvature & $\mathrm{m}^{-2}$ \\
\hline$H$ & Mean curvature & $m^{-1}$ \\
\hline$R$ & Cylinder radius & $\mathrm{m}$ \\
\hline$\alpha$ & Liquid volume fraction & - \\
\hline$\Sigma$ & Mean surface density & $m^{-1}$ \\
\hline$\kappa$ & Principal curvature & $\mathrm{m}^{-1}$ \\
\hline$\varphi$ & Distance function from the interface & $\mathrm{m}$ \\
\hline$\rho$ & Density & $\mathrm{kg} \cdot \mathrm{m}^{-3}$ \\
\hline$\mu$ & Viscosity & $\mathrm{kg} \cdot \mathrm{m}^{-1} \cdot \mathrm{s}^{-1}$ \\
\hline$\sigma$ & Surface tension & $\mathrm{kg} \cdot \mathrm{s}^{-2}$ \\
\hline acronym & definition & \\
\hline DNS & Direct Numerical Simulation & \\
\hline DSD & Drop Size Distribution & \\
\hline $\mathrm{ICM}$ & Interface Capturing Method & \\
\hline LES & Large Eddy Simulation & \\
\hline NDD & Number Diameter Distribution & \\
\hline RANS & Reynolds Averaged Navier-Stokes & \\
\hline $\mathrm{SCD}$ & Surface Curvature Distribution & \\
\hline CLSVOF & Coupled Level Set/Volume Of Fluid & \\
\hline
\end{tabular}

Table 1: Nomenclature and acronyms 


\section{Introduction}

Liquid fuel atomization and its subsequent combustion nowadays still represents one of key source for energy production both in aero-engine and automotive context. Considering the difficulties and the high costs of experimental

5 measurements for two phase flow mainly in the dense spray region, in last years numerical simulations have been reliably used to gain a detail insight in liquid evolution. Several numerical methods, associated to different computational costs, have been therefore developed to this end (see for instance Gorokhovski and Herrmann (2008); Demoulin et al. (2013) and references therein) and they are normally aimed at providing predictive tools to be used to understand the various physical mechanisms involved in the atomization context.

A standard output normally expected from any atomization model or theory, going from DNS studies till experimental correlations is the drop size distribution (DSD). From a theoretical point of view, several definitions of this function can be introduced. Generally, it is $F_{N}(D)$ in such way that $F_{N}\left(D^{\prime}\right) d D$ is the number of droplet per unit of volume with a diameter $\in\left[D^{\prime}, D^{\prime}+d D[\right.$. In this case, it is the number diameter distribution (NDD) and it may be also normalized to define a probability density function $P_{N}(D)$. The function $F_{N}(D)$ requires the possibility to count the number of droplets. Thus, it is necessary to separate the liquid phase in a set of discrete elements. However, usual atomization process starts with a continuous liquid flow (for instance a liquid jet) and during the atomization a splitting of the continuous liquid phase occurs. Once it is created, for fixed external conditions and generally considering the whole spray, the NDD may evolve towards an asymptotic state, for which numerous Sojka, 2002). To address more complex situations or to determine its function in space and time, a transport equation for the DSD, like the Williams Boltzmann Equation (WBE) (Williams, 1958), is required.

Although the flow inside the injector as well as surface instabilities and the methods to solve the WBE have been the subject of several research works 
in technical literature, few studies deal with the droplet generation step to estimate the DSD. One reason is the lack of data to justify any proposal as well as the absence of a definition of the NDD during the primary breakup. In-fact, considering a liquid jet before the detachment of a liquid parcel, the notion of a diameter cannot be reasonably introduced. Nonetheless, considering an initial instability over a liquid surface, such unsteadiness should evolve in time and create wrinkles at a certain length scale that is somehow related on a theoretical point of view to the diameter of the droplet that will be then generated. It is clear that a link between these two subsequent steps is missing. Regarding the data in this transition zone, lately there have been great progresses both in experimental techniques (Déjean et al., 2016; Warncke et al., 2017) and in numerical simulations (Navarro-Martinez, 2014, Dumouchel et al., 2017, Fox, 2012). However, an extended definition of the DSD should be first introduced to be able to deal with the dense spray region as well as with primary atomization.

45 It should be pointed out that the same problem appears in defining a mean diameter: it is a moment of the NDD and therefore it can be defined only once the continuous liquid jet has been broken in a set of liquid droplets.

The main goal of the present study is therefore the proposition of an innovative extended definition of the drop size distribution to be used all along the atomization process, leading to the so called Surface Curvature Distribution (SCD). In the following, the definition of the SCD will be firstly introduced and related to some characteristic geometrical properties of the liquid/gas interface, such as the mean $(\mathrm{H})$ and Gauss $(\mathrm{G})$ curvatures. Then, numerical simulations will be used to extract the evolution of such geometrical properties on fundamental test cases. The well-known Archer code (Tanguy and Berlemont, 2005. Ménard et al., 2007), where a combined VOF-Level set approach is used to capture the interface and a ghost-fluid method is applied to represent accurately the jump of variable across the liquid-gas interface, has been used to this end. A database of DNS data focused on interfacial geometrical quantities will 6o be presented to provide valuable information to improve atomization modeling in particular for the Eulerian Lagrangian Spray Atomization (ELSA) model 
(Lebas et al., 2009, Chesnel et al., 2011) in the region where the transition to a Lagrangian frame for the liquid phase is realized.

The paper is structured as follows: the next section is devoted to the descrip-

65 tion of the SCD and to the introduction of a relation between the SCD and the NDD for a spherical droplet based on surface curvatures. In the second part of the work, a Rayleigh-Plateau and a droplet collision test case will be presented to characterize the two curvatures and to show how the breakup/coalescence process from a liquid jet to the final droplet can be directly described employing such surface topological properties. Finally, an Homogeneous Isotropic Turbulence test case will be considered and the evolution of $\mathrm{H}$ and $\mathrm{G}$ addressed for several values of liquid volume fraction in order to mimic the atomization process.

\section{An extended definition of the drop size distribution}

The purpose of this section is the proposition of an extended definition of the drop size distribution that may applicable all along the atomization process including the primary breakup zone. From the pioneering work of Vallet and Borghi (1999); Vallet et al. (2001), it is known that the definition of the mean diameter can be replace beneficially by the more general mean surface density $\Sigma$, that is the surface area per unit of volume. This quantity can be defined everywhere whatever is the liquid phase topology and, combined with the liquid volume fraction $\alpha$, it gives the mean Sauter diameter once the spray is formed: $D_{32}=\frac{6 \alpha}{\Sigma}$. Recently, Essadki et al. (2018a) used high order fractional moments of the DSD for disperse phase, where the size is given by the surface area of droplet, to recover some interface geometrical quantities already used in describing the gas-liquid interface in their previous work (Essadki et al., 2016). These quantities are the volume fraction, the mean surface density and the two averaged Gauss $G=\kappa_{1} * \kappa_{2}$ and mean $H=\frac{\kappa_{1}+\kappa_{2}}{2}$ curvatures, where $\kappa_{1}$ and $\kappa_{2}$ are the two principal curvatures of the surface.

To overcome the limits of the DSD in modeling a gas-liquid interface during 
the whole atomization process, the first idea here proposed is the exploitation of some surface characteristics to look at the DSD since they carry the information of the size distribution which is hidden all along the wrinkling process. The proposal is to look at the curvature $(\kappa)$ distribution. In this case the distribution 95 of curvature is $F_{S}(\kappa)$ and the $F_{S}\left(\kappa^{\prime}\right) d \kappa$ is the area of surface with a curvature $\kappa \in\left[\kappa^{\prime}, \kappa^{\prime}+d \kappa[\right.$. This leads to the definition of a surface curvature distribution (SCD). However, an accurate description of the interface cannot be restricted to one geometrical variable as it is often used in the DSD for disperse phase. Therefore, the Gauss and mean curvatures are proposed here as phase-space variables, leading to the definition of the novel bi-variate SCD: $F_{S}(H, G) . H$ and $G$ are much more meaningful to be used in this context with respect to $\kappa_{1}$ and $\kappa_{2}$. Indeed, the curvatures $\kappa_{1}$ and $\kappa_{2}$ need to be distinguished by considering an order relation $\kappa_{1} \leq \kappa_{2}$, while the curvatures $H$ and $G$ are mathematically well defined and provide some interesting topological properties as proposed in Essadki et al. (2018b).

The definition of $F_{S}(H, G)$ and its general link with the standard number density function is based on the introduction of the Gauss-Bonnet theorem ( See Essadki et al. (2018b) for details). Considering $M$ as a 3D object, delimited by a surface $\Sigma(M)$, the following relation stands:

$$
\int_{x \in \Sigma(M)} G(x) d S(x)=2 \pi \chi(M)
$$

where $\chi(M)$ is the Euler characteristic that is equal for any homeomorph object to M. Such quantity is particularly meaningful since for instance it is equal to 2 for objects homeomorph to a sphere, considering a set of droplets homeomorph to a sphere, it can be then used to count the number of particles. In the case 110 of a sphere $\kappa_{1}=\kappa_{2}=\frac{2}{D}$ and the Gauss curvature can be simply expressed as $G=\frac{4 \pi}{S}$, where $S=\pi D^{2}$ is the droplet surface area. Hence, the two functions $\frac{G F_{S}(H=\sqrt[2]{G}, G)}{4 \pi}$ and $F_{D}(D)$ are equivalent, since they count the number of droplets per volume and per radius or Gauss curvature. The demonstration of this equivalence between the two distributions in a more general case can be 115 found in Essadki et al. (2018b). In Figure 1, the link between the DSD and for 
instance the probability distribution of $H$ is shown for a cloud of droplets with a known normal distribution (i.e. $D_{m e a n}=5 \mathrm{e}-05 \mathrm{~m}$ and $\sigma=1 \mathrm{e}-05$ ). A continuous link between the two distributions can be introduced and the DSD can be easily recast starting from the corresponding curvature values. Nonetheless, the SCD is defined all along the atomization process and therefore, once the spray is formed, the DSD is a particular case of $F_{S}(H, G)$.

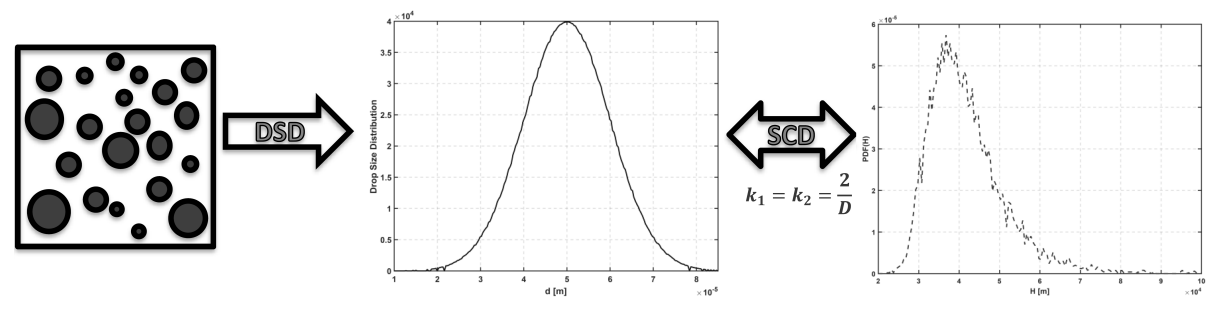

Figure 1: Link between the DSD and the SCD for a known cloud of spherical droplets.

We should mention that the SCD is a distribution that measures the probable surface area having some given interfacial properties. As a matter of fact, it can not cope with the volume occupied by the liquid or the gas phase. Another approach to characterized the spray consists in using a volumetric distribution. For a spray, the DSD becomes $F_{V}(D)$ in such way that $F_{V}\left(D^{\prime}\right) d D$ is the liquid volume fraction of droplets with a diameter $\in\left[D^{\prime}, D^{\prime}+d D[\right.$. This volumetric distribution can be also extended to any liquid topology, leading to the volumetric distance function distribution $(V \varphi D)$, that can be a second proposal to describe the transport of curvatures. It is defined by $F_{V}(\varphi)$ in such way that $F_{V}\left(\varphi^{\prime}\right) d \varphi$ is the volume fraction of flow that is at a distance $\varphi$ from the interface, with $\varphi \in\left[\varphi^{\prime}, \varphi^{\prime}+d \varphi[, \varphi\right.$ is positive in the liquid phase and negative in the gas phase. With this definition the following relation stands: The gas volume fraction is $\alpha_{g}=\int_{-\infty}^{0} F_{V}(\varphi) d \varphi$, the liquid volume fraction is $\alpha_{l}=\int_{0}^{+\infty} F_{V}(\varphi) d \varphi$, and the mean surface density $\Sigma=F_{V}(0)$.

The $V \varphi D$ gives the distribution of the flow with respect to the distance to the interface. The link with the DSD is not straightforward. However, 
this distribution can be clearly identified on a given geometric object and in particular for a spherical droplet.

A similar idea has been also explored by Dumouchel et al. (2015, 2017) with the concept of scale distribution, $E_{3}(d)$. The definition of this function for a given object (for instance a droplet) is based on the total object volume $V_{0}$ and the volume $V(d)$ defined by all points at a distance $d$ or greater to the object surface. The scale distribution is given by $E_{3}(d)=\frac{V_{0}-V(d)}{V_{0}}$. The link between the scale distribution and the $V \varphi D$ can be express readily by $E_{3}(d)=\frac{F_{V}(\varphi=d)}{\alpha_{l}}$ Dumouchel et al. (2015) have worked on experimental data in $2 \mathrm{D}$ and more recently they have used numerical simulations to extract $3 \mathrm{D}$ results (Dumouchel et al. 2017). A connection with curvatures is surely present but further investigations are required on this point.

Following the idea of Essadki et al. (2018b), the emphasis of this work is to analyze the surface curvature distribution and the curvature behavior (Gauss, mean and principal curvatures) in multiple two-phase flows configurations. These quantities give an accurate geometric description of the interface behavior. This article aims to propose a new framework to study the atomization process, based on the curvature and SCD time evolution.

\section{Governing equations and numerical methods}

\subsection{Navier-Stokes equations and interface capturing method}

Here, a joint level set/VOF method are coupled with a projection method to carry out the direct numerical simulation of incompressible Navier-Stokes equations:

$$
\frac{\partial \boldsymbol{V}}{\partial t}+(\boldsymbol{V} . \nabla) \boldsymbol{V}=-\frac{\nabla p}{\rho}+\frac{1}{\rho} \nabla \cdot(2 \mu \boldsymbol{D})+\boldsymbol{f}+\frac{1}{\rho} \sigma H \delta(\varphi) \boldsymbol{n}
$$

where $p$ is the fluid pressure, $\boldsymbol{V}$ is the velocity vector, and $\boldsymbol{D}$ is the viscous deformation tensor. At the interface, the surface tension force can be considered based on the Dirac function $\delta(\varphi)$. Concerning the interface capturing method, a CLSVOF interface capturing method is used to ensure mass conservation and 
165 a accurate description of the interface topology (See Ménard et al. (2007) for details).

This work benefits for the latest improvements of the ARCHER code : the convective term is solved using the improved Rudman technique presented in Vaudor et al. (2017), allowing a better accuracy and description of high density

170 ratio flows. This method is based on the computation of mass fluxes from the VOF method, which can then be used in a conservative formalism of the convective term. The diffusive term is now computed with the approach of Sussman et al. (2007), the main advantage of this method is the implicit jump condition of the viscous tensor. For further details, a review of the numerical methods dedicated to the computation of the viscous term in two-phase incompressible flows is available in Lalanne et al. (2015).

Fluid dynamics equations are solved in the context of a low Mach number approach, based on a projection method for the direct numerical simulation of incompressible Navier-Stokes equations (detailed in Vaudor et al. (2017)). The viscosity depend on the sign of the level set function according to each phase (liquid and gas). To finalize the description of the two-phase flow, jump conditions across the interface are taken into account with the ghost fluid (GF) method. In the GF method, ghost cells are defined on each side of the interface Kang et al. (2000); Liu et al. (2000)). This prolongs each phase to allow smooth derivatives in the vicinity of the interface. As defined previously, the interface is characterized through the distance function, and jump conditions are extrapolated on a few nodes on each side of the interface.

\subsection{Curvature computation}

Two methods have been used to extract the curvature distribution. The first one is based on the distance function $(\varphi)$ that is part of the numerical procedure in the resolution of the two-phase flow Navier-Stokes equations. The normal to the surface $\vec{n}=-\frac{\vec{\nabla} \varphi}{|\vec{\nabla} \varphi|}$ is first calculated. Then, the matrix $P=I-\vec{n} \vec{n}^{T}$ with $I$ the identity matrix, and the Hessian matrix $H_{m}=\frac{\partial^{2} \varphi}{\partial x_{i} \partial x_{j}}$ are calculated in order to have the matrix $G_{m}=-\frac{P H_{m} P}{|\vec{\nabla} \varphi|}$. Finally, the two principal curvatures 
$195 \kappa_{1}$ and $\kappa_{2}$ are obtained as $\kappa_{1}=\frac{T+\sqrt[2]{2 F^{2}-T^{2}}}{2}$ and $\kappa_{2}=\frac{T-\sqrt[2]{2 F^{2}-T^{2}}}{2}$ with $T$, the trace of the matrix $G_{m}$ and $F$, the Frobenius norm of the matrix $G_{m}$. The reader interested in a more detailed explanation of such method is addressed to Kindlmann et al. (2003). The second one has been instead developed in Essadki et al. (2018b), where the gas-liquid interface is discretized with a $2 \mathrm{D}$ triangulated mesh using the Marching Cube algorithm. The curvature is computed at each vertex of the generated mesh through a spatial-averaging process that preserves some topological feature of the gas-liquid surface such as the Gauss-Bonnet formula (Meyer et al. 2002). The topological features of the interface in terms of computing the droplet number from the bi-variate SCD, while filtering the noise, are in this way guaranteed. On the other hand, results for the $V \varphi D$ have been obtained directly through the distance function.

\section{Results and discussion}

This section is dedicated to the analysis of the curvature behavior and statistics in two different configurations. First, a Rayleigh-Plateau instability and a droplet collision configurations are investigated to illustrate our analysis on simple validation cases. Then, a more complex configuration closer to the atomization regime (high Weber and Reynolds number) presenting liquid structures of various shape and size is studied. Namely, the two-phase flows Homogeneous Isotropic Turbulence configuration.

\subsection{Rayleigh-Plateau instability}

\subsubsection{Numerical configuration}

As a first configuration, the Rayleigh-Plateau instability, where a set of droplets is created from an initial column of liquid, is reported. A quarter of a cylinder has been here computed and symmetric boundary conditions have been

220 employed in a computational box of $1,5 \cdot 10^{-4} \times 1,0.10^{-4} \times 1,0.10^{-4} \mathrm{~m}$ length. A mesh sizing of $96 \times 64 \times 64$ elements as in Ménard (2007) has been chosen. This is a standard test case for the ARCHER code that is able to correctly reproduce 
the dispersion analysis diagram on this configuration (Ménard, 2007). The liquid and gas properties are reported in Table 2.

\begin{tabular}{|c|c|c|c|c|}
\hline $\begin{array}{c}\rho_{l} \\
\mathrm{~kg} \cdot \mathrm{m}^{-3}\end{array}$ & $\begin{array}{c}\mu_{l} \\
\mathrm{~kg} \cdot \mathrm{m}^{-1} \cdot \mathrm{s}^{-1}\end{array}$ & $\begin{array}{c}\rho_{g} \\
\mathrm{~kg} \cdot \mathrm{m}^{-3}\end{array}$ & $\begin{array}{c}\mu_{g} \\
\mathrm{~kg} \cdot \mathrm{m}^{-1} \cdot \mathrm{s}^{-1}\end{array}$ & $\begin{array}{c}\sigma \\
\mathrm{kg} \cdot \mathrm{s}^{-2}\end{array}$ \\
\hline 1000 & $1,0.10^{-3}$ & 1.0 & $1,879 \cdot 10^{-3}$ & 0,072 \\
\hline
\end{tabular}

Table 2: Physical properties of the Rayleigh Plateau instability

225

The cylinder has a radius $R=3 \cdot 34 \cdot 10^{-5} \mathrm{~m}$ and the initial perturbation, essential to observe the instability, has an amplitude of $10 \%$ of the radius of the cylinder and a wavelength of $R=3 \cdot 0 \cdot 10^{-4} \mathrm{~m}$ (twice the length of the box). The initial conditions have been chosen in order to have a wave number satisfying $k_{w} R=0.7$ which correspond to the fastest growth rate.

\subsubsection{Comparison between both curvature evaluation method}

Both method aforementioned to extract the curvature distribution are tested on one time frame of the Rayleigh-Plateau test case, but no significant differences are observed as shown in Figure 2. The mean and Gauss curvatures are provided as dimensionless using the radius of the initial cylinder as reference length, whereas for the Gauss curvature the radius squared has been used to this end. Considering that the two approaches behave in a completely consistent manner, in the following all results have been obtained using Method 1. The parabola represents the spherical shape (i.e. $k_{1}=k_{2}$ ) and the dotted black line is the cylinder $(\mathrm{G}=0)$.

\subsubsection{Curvature evolution and statistics}

To have a deep insight in the evolution of the breakup event, four time frames have been selected and are shown in Figure 3. It is possible to see the initial configuration $\left(t_{a}\right)$, the deformation of the cylinder with the generation of the bottle-neck $\left(t_{b}\right)$, the moment of the break-up $\left(t_{c}\right)$ and the final generation

245 of droplets $\left(t_{d}\right)$. It is interesting to analyze the evolution of surface curvatures during this breakup process. Five different representations have been realized to 

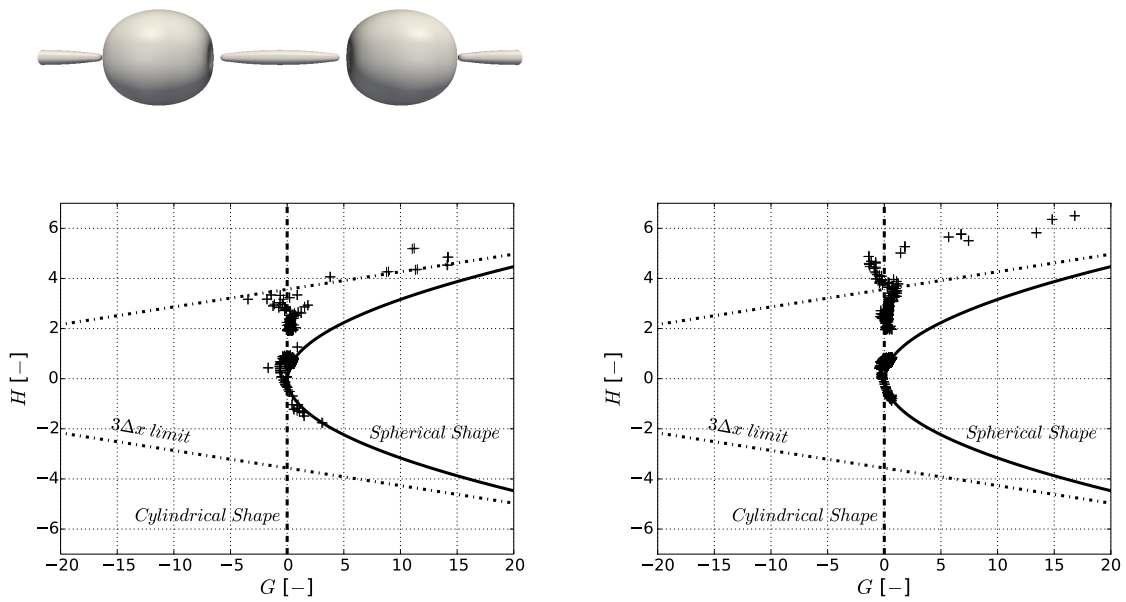

Figure 2: Rayleigh Plateau instability at $t=1,88 \cdot 10^{-4}$ (Top) and mean curvature in function of Gauss curvature with the first method (left) and with the second method (right).

study the evolution of curvature ; a graph showing mean curvature $H$ in function of Gauss curvature $G$ (Figure 4 top-left), an other showing the two main curvatures $\kappa_{1}$ and $\kappa_{2}$ (Figure 4 top-right). For completeness, various kinds of representation can be used and are also mentioned in this paper : a graph showing $H$ in function of $\sqrt{|G|}$ (Figure 4 center-left) in order to have variables with the same physical dimension, and a graph $S$ in function of $P$ (Figure 4 centerright) showing the dual space of $H$ and $G$ graph. $S$ represents $\frac{R_{1}+R_{2}}{2}$ and $P$ represents $R_{1} R_{2}$ with $R_{1}=\frac{1}{\kappa_{1}}$ and $R_{2}=\frac{1}{\kappa_{2}}$, the two curvature radii. The final graph represents the $\left(\kappa_{1}-\kappa_{2}, \kappa_{1}+\kappa_{2}\right)$ space which is the $\left(\kappa_{1}, \kappa_{2}\right)$ graph with a $45^{\circ}$ rotation. The dashed lines correspond to a cylindrical topology ; it means that one of the two main curvatures is equal to zero and therefore the Gauss curvature $G$. The solid lines correspond to a spherical topology so $\kappa_{1}=\kappa_{2}$. On the $(H, G)$ and $(S, P)$ graphs, it forms a parabola and no points can be inside this parabola. By the way it is calculated, $\kappa_{1}$ is always greater than $\kappa_{2}$ so $\kappa_{1}-\kappa_{2}$ is always positive. On the $\left(\kappa_{1}-\kappa_{2}, \kappa_{1}+\kappa_{2}\right)$ graph, the dash-dotted line separates "bubbles" on the left from "droplets" on the right. 
Besides, on the $(H, G)$ and $(S, P)$ graphes, the limits of the mesh resolution are also represented by dotted lines. In the following, only the $(H, G)$ graph and the $\left(\kappa_{1}-\kappa_{2}, \kappa_{1}+\kappa_{2}\right)$ graph are conserved, mainly due to their mathematical properties (see Section 2). On Figure 9 and 10 , the distance function $\varphi$ is also reported to better understand the obtained results and it is dimensionless using $d x$ as reference and the curves are shown between $-l_{x}$ and $l_{x}$.

As long as the cylinder is weakly deformed (Figure 9), in terms of PDF profile for $\varphi$, points are very close to the theoretical shape of a cylinder with a linear curve inside the liquid part. In the figure, line without markers represents the initial cumulative profile for a cylinder, whereas the dashed line separates liquid (positive values) and gas (negative values). For the cumulative of total curvature (i.e. 2H) in Figure 8-left and Gauss curvature in Figure 7-left, a step profile at values corresponding to this cylinder is derived. On the graph showing mean curvature in function of Gauss curvature (Figure 5-top-left), all the points are at this time step all gathered around the vertical line at $\mathrm{G}=0$.

Then, when such cylinder is deformed $\left(t_{b}\right)$, negative values for Gauss curvature appear (Figure 7 right) related to all the saddle points in the middle of the cylinder. This portion of the cylinder is visible on the mean and Gauss curvatures graph (Figure 5-top-right) with corresponding points that create an arc. Besides, on this graph, the points, which represent the big part of the cylinder, get closer to the parabola representing the sphere.

When the break-up happens $\left(t_{c}\right)$, such arc breaks up and two clouds of points appear (see Figure 5-bottom-left) with one set located on the black curve. These points correspond to the big droplet. Indeed, the mean curvature is approximately 0.5 and the Gauss curvature about 0.25 which correspond to a radius twice as big as the initial jet radius. The other points, located below the parabola, correspond to the small ellipsoid. Here, the mean curvature is quite high because of the small thickness and the Gauss curvature is lower because the curvature is close to zero along the length. The big droplet appears also as the step on the total curvature cumulative shown in Figure 8-right and the small ellipsoid by all the high positive values. Such break-up process is mainly 

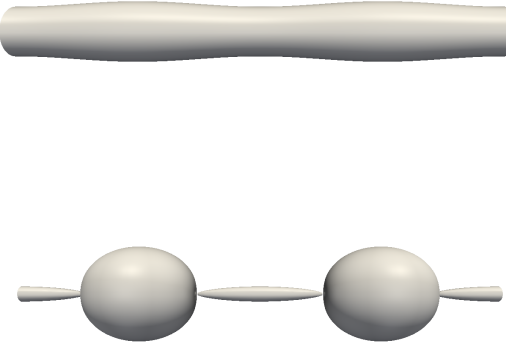
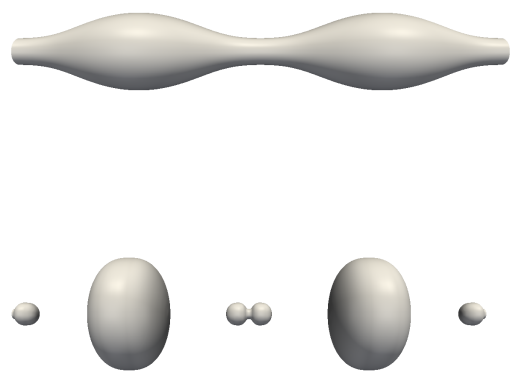

Figure 3: Top left : Rayleigh Plateau instability at $t_{a}=3,92.10^{-7} s$; Top right : Rayleigh Plateau instability at $t_{b}=1,49.10^{-4} s$; Bottom left : at $t_{c}=1,86 \cdot 10^{-4} s$; Bottom right : at $t_{d}=2,03 \cdot 10^{-4} s$

evident on the curvature plot since small variations on the interface geometry can lead strong changes in the curvature values.

Finally, when the two droplets are generated $\left(t_{d}\right)$, for the mean and Gauss curvatures graph (see Figure 5-bottom-right) most of the points are on the spherical curve. The points corresponding to the big droplet are located approximately at the same place than the previous time. Conversely, points corresponding to the small droplet are those having an higher curvature. On Figure 10, PDF profile becomes now parabolic in the liquid part, as expected for spheres, with higher values of the distance function because the generated droplet is bigger than the initial cylinder. On the $\left(\kappa_{1}-\kappa_{2}, \kappa_{1}+\kappa_{2}\right)$ graph (Figure 6), same comments than on the $(H, G)$ graph can be done ; the arc when the cylinder is deformed, the two clouds of points when the break-up happens and the location of the points with respect to the spherical and cylindrical shapes.

Hence, the analysis of the evolution of Gauss and mean curvatures can be reliably employed to describe a breakup process from a liquid jet to the final droplet. Distributions of $H$ and $G$ follow the physical process here investigated suggesting that their evolution all along the atomization process can be characterized. 

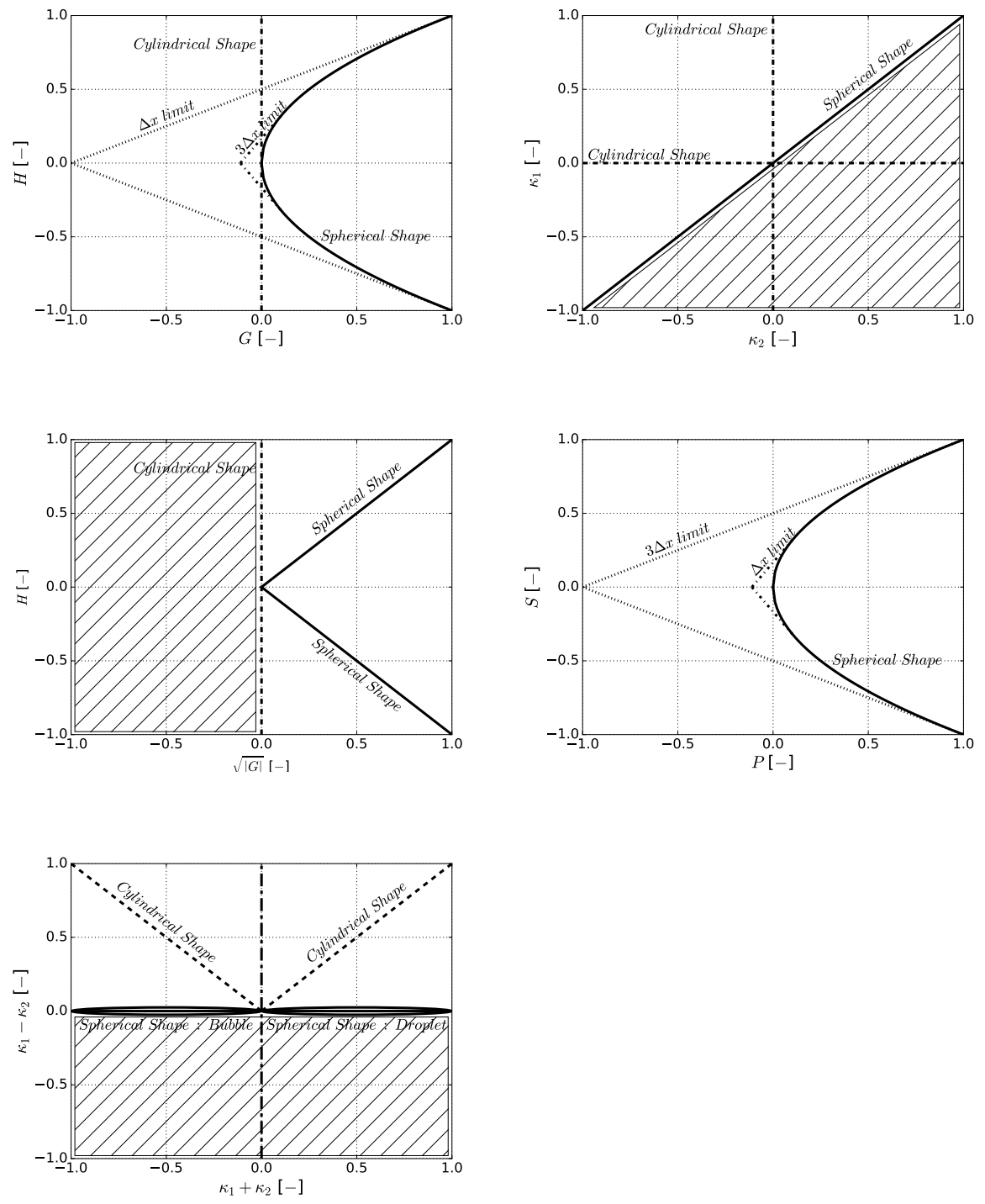

Figure 4: Different spaces for curvature representation. 

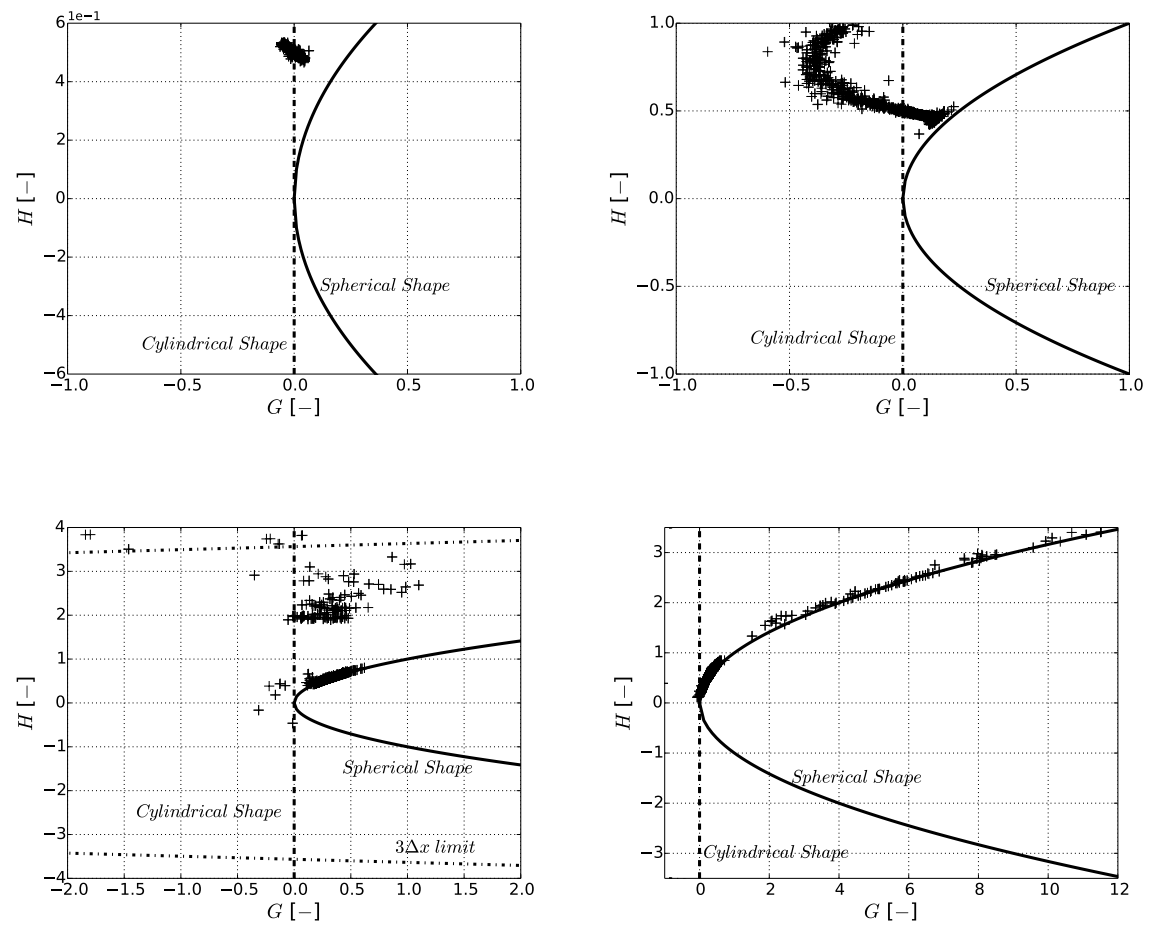

Figure 5: Mean curvature in function of Gauss curvature (from top left to bottom right from $t_{a}$ to $\left.t_{d}\right)$.The solid line represents a spherical shape $\left(\kappa_{1}=\kappa_{2}\right)$ and the dashed line represents a cylindrical shape $(G=0)$. The dash-dotted line on $t_{c}$ represents the resolution limit $3 \Delta x$.

\subsection{Droplet collision}

\subsubsection{Numerical configuration}

The capabilities of such geometrical surface properties have been also tested for a collision test case, which represents another fundamental step in the deformation of liquid surface. The ARCHER code has been already widely validated also on this configuration (Tanguy and Berlemont, 2005; Luret et al., 2010) and here a test point with two spherical water droplets of initial radius of 200 $\mu m$ and $130 \mu m$, characterized by an impact factor $\mathrm{I}=0.35$, a relative velocity $V_{\text {coll }}=3.42 \mathrm{~ms}^{-1}$, a Weber liquid number $W e_{l}=33.5$ and an Ohnesorge of $O h=0.015$ has been considered. 

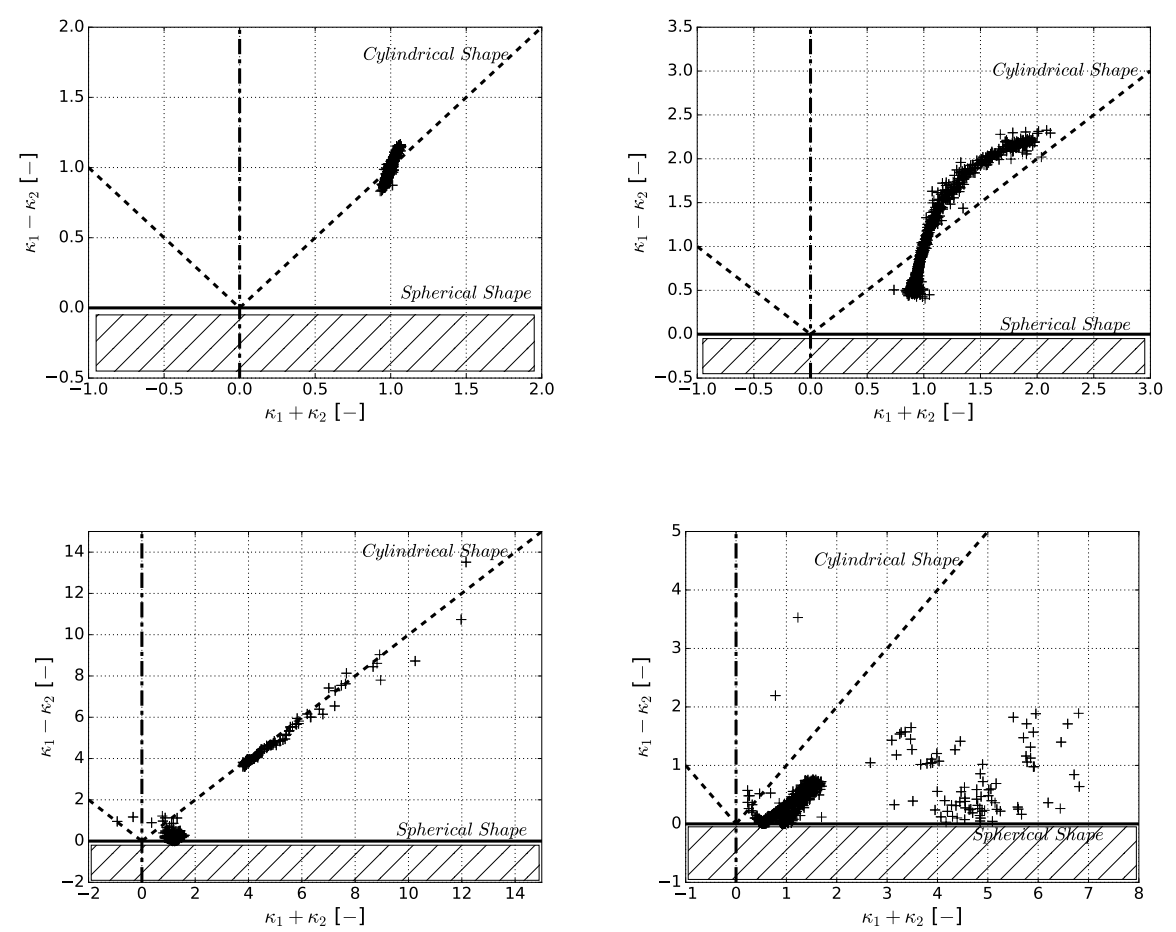

Figure 6: Mean curvature in function of Gauss curvature (from top left to bottom right from $t_{a}$ to $\left.t_{d}\right)$. The dash-dotted line represents the separation between droplets and bubbles and the dashed lines represent a cylindrical shape $(\kappa=0)$. The solid line represents a spherical shape $\left(\kappa_{1}=\kappa_{2}\right)$.

\subsubsection{Curvature evolution and statistics}

In Figure 11 the resulting evolution of the liquid surface on four time frames is shown, where it should be pointed out the initial configuration $\left(t_{a}\right)$, the moment of coalescence $\left(t_{b}\right)$, the generation of one deformed droplet $\left(t_{c}\right)$ and its subsequent breakup with the generation of a torus structure $\left(t_{d}\right)$. In Figure 12 the corresponding evolution on the $(H, G)$ plane is reported, where it can be pointed out that, similarly to the Rayleigh-Plateau test case, the curvatures evolve consistently with the investigated physical process.

Considering this preliminary assessment on fundamental test cases, in the next section, the same surface geometrical properties will be used to analyze a 

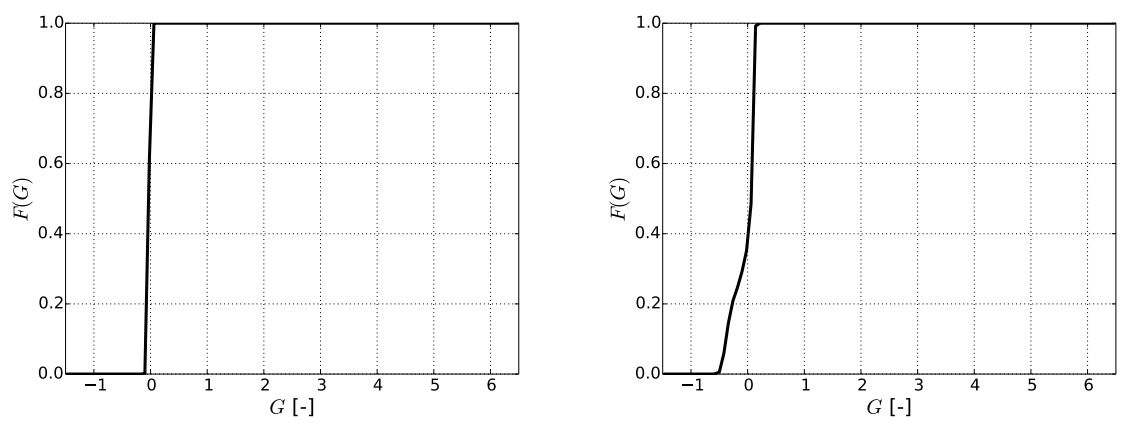

Figure 7: Cumulative of the Gauss curvature (Left : at $t_{a}$; Right : at $t_{b}$ )
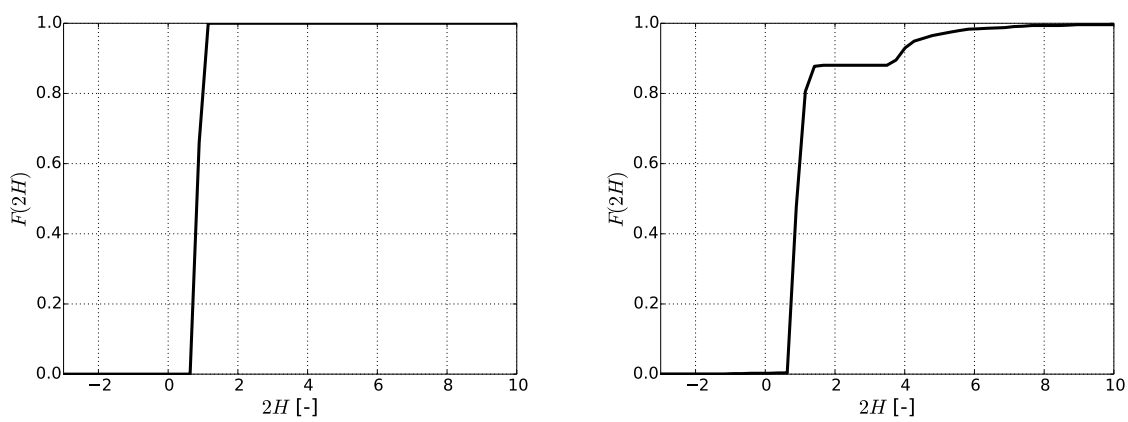

Figure 8: Cumulative of the total curvature (Left : at $t_{a}$; Right : at $t_{c}$ )

Homogeneous Isotropic Turbulence (HIT) box where breakup and coalescence events appear together due to the turbulence forcing.

\subsection{Homogeneous Isotropic Turbulence configuration}

\subsubsection{Numerical configuration}

A further assessment and exploitation of the procedure regarding curvature extraction has been performed using a three dimensional cubical domain with periodic boundaries similar to the investigation of Duret et al. (2012, 2013). This configuration represents a extension of single phase mixing studies to two phase flow and it has been already analyzed to improve the understanding of primary atomization (see Duret et al. $(2012,2013)$ ). [h] 

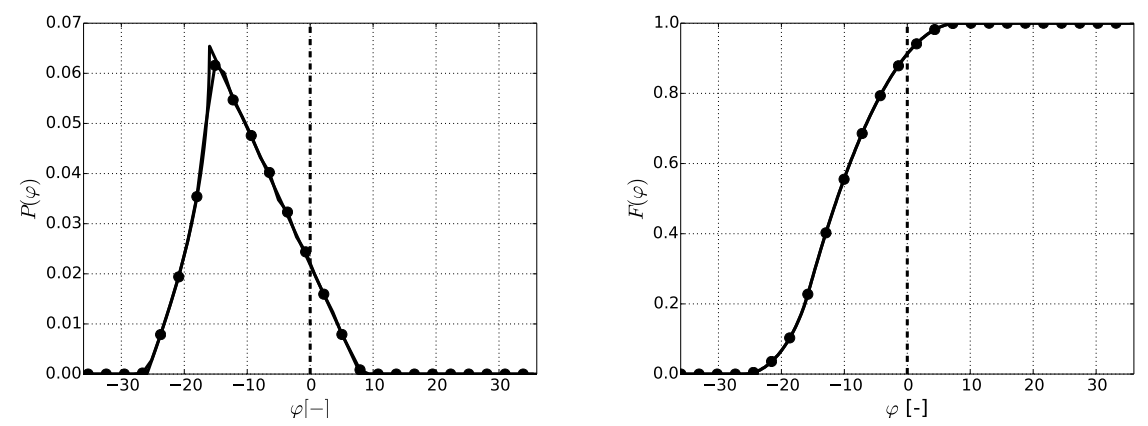

Figure 9: Left : PDF of the distance function at $t_{a}$; Right : cumulative of the distance function at $t_{a}$. The lines with $\bullet$ represent the numerical results, the solid lines represent the theoretical shape for a cylinder and the dashed lines separate gas (on the left) from liquid (on the right)

\begin{tabular}{|c|c|c|c|c|c|}
\hline$\rho_{l} / \rho_{g}$ & $\sigma$ & $\mu_{g}$ & $\mu_{l}$ & $\bar{k}_{k i n}$ & $\mathrm{~L}$ \\
\hline 30 & 0.0135 & $1.879 \mathrm{e}-05$ & $5.65 \mathrm{e}-04$ & 3.6 & $1.5 \mathrm{e}-04$ \\
\hline
\end{tabular}

Table 3: Properties for DNS simulations of HIT (S.I. units).

The mesh resolution used in the present investigation is based on previous studies on the same configuration and on single-phase flow simulations to capture the Kolmogorov length scale (Duret et al., 2012, 2013). In Duret et al. (2013),

345 the fine computational domain counting $256^{3}$ mesh elements has been employed. The validation of this set-up for single-phase simulation is not here shown for the sake of clarity. The interested reader is addressed to Duret et al. (2012), where this topic is discussed in detail.

In order to define a configuration with realistic interactions between the liquid surface and the turbulent gas flow-field, the following dimensionless parameters have been selected (i.e. coherently with Duret et al. (2013)): gaseous Weber number $W e_{g}=\rho_{g} \bar{k}_{k i n} L / \sigma=1$, liquid Weber number $W e_{l}=\rho_{l} \bar{k}_{k i n} L / \sigma=30$, liquid based Reynolds number $R e_{l}=\sqrt{\bar{k}_{k i n}} L / \nu_{l}=310$ and liquid Ohnesorge number $O h_{l}=\sqrt{W e_{l}} / R e_{l}=1.77 e-02$, where $\bar{k}_{k i n}$ is the mean kinetic energy, 

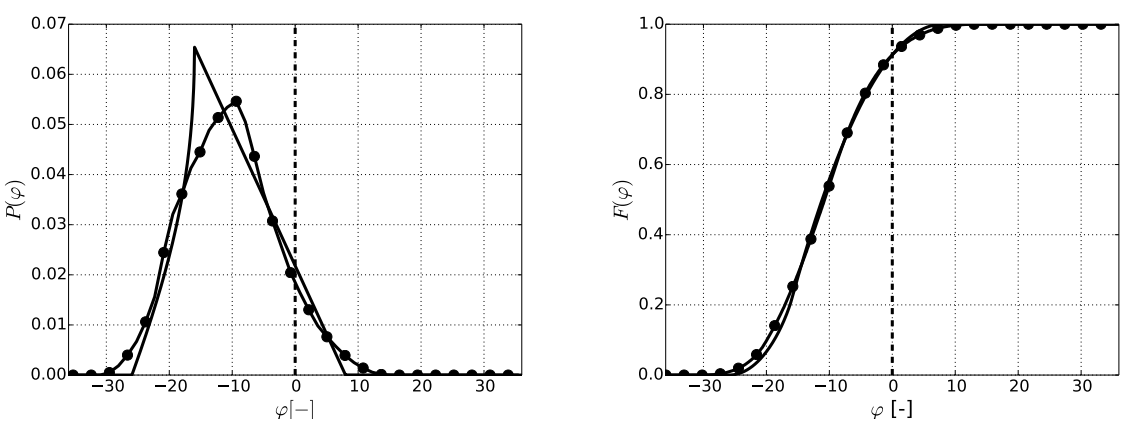

Figure 10: Left : PDF of the distance function at $t_{d}$; Right : cumulative of the distance function at $t_{d}$. The lines with $\bullet$ represent the numerical results, the solid lines represent the theoretical shape for a cylinder and the dashed lines separate gas (on the left) from liquid (on the right)

$\sigma$ the surface tension, $\nu$ the kinematic viscosity in liquid or gas phase, $\rho$ the density and $L$ is the length of the box. In Table 3 the corresponding set of physical properties is reported.

Several values of the liquid volume fraction $\alpha_{l}$ inside the box have been studied in the present work for curvature extraction (i.e. ranging from $1 \%$ to $90 \%$ ) in order to mimic the whole atomization process as shown in Figure 13 using the data obtained from DNS calculation on a diesel jet. The main aim is the extraction of curvatures evolution all along the jet evolution in order to point out firstly the link between the DSD and the $F_{S}(H, G)$ and also how curvatures evolve from the dense region up to the dispersed spray (i.e. reducing $\alpha_{l}$ ).

\subsubsection{Curvature evolution and statistics}

Results obtained for the HIT varying $\alpha_{l}$ are here presented starting from the $1 \%$ test case, which can be reliably considered as representative of the dilute spray region. A snapshot of the liquid-gas interface distribution together with the evolution in time of $\bar{\Sigma}$ and $\bar{H}$, which represent the volume average for the whole domain of liquid-gas interface and mean curvature, as well as their time averaged values is reported in Figure 14. In the following, $t^{*}$ is the time 

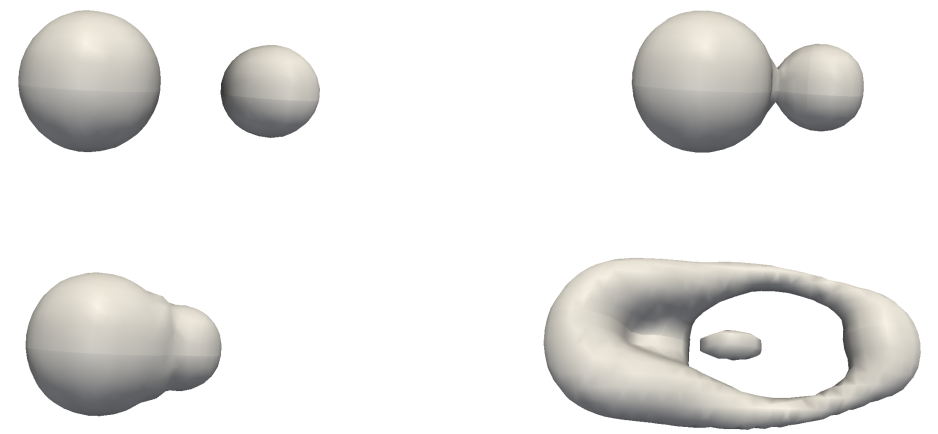

Figure 11: Top left : Surface evolution for droplet collision test case ; Top left : at $t_{a}=$ 2.97.10 $0^{-5} s$; Top right: at $t_{b}=7 \cdot 2 \cdot 10^{-5} s$; Bottom left : at $t_{c}=9 \cdot 9 \cdot 10^{-5} s$; Bottom right : at $t_{d}=4.3562 .10^{-4} s$

normalized by the turbulent time scale of the box, i.e. $\tau_{t}=\frac{\bar{k}_{k i n}}{\epsilon}$.

It is possible to point out the augmentation and reduction of $\Sigma$ in time related to breakup or coalescence events as well as the generation of a cloud of nearly spherical droplets from the instantaneous time frame. The characteristic sizing of the generated liquid structures covers a wide range of diameters: big droplets, which carry the major part of the total mass, can be clearly identified and from their turbulent collisions smaller particles are generated. Clearly, with respect to the Rayleigh-Plateau test case, the representation of the breakup process is here much more complex due to the local appearance of several different values of the surface curvatures. Several nearly spherical droplets are generated together with some liquid structures characterized by high negative Gauss curvature values leading to a non-straightforward discussion of physical phenomena on the $(H, G)$ graph used in previous section.

Therefore, in order to have a deeper insight into the evolution of surface characteristics in this test case, it has been chosen to analyze some statistical properties of $H$ and $G$. In particular, in Figure 16 the time averaged PDF of the mean curvature is shown. Such time averaged distribution has been obtained by dividing the $H$-space in a user-defined number of classes and by collecting during the cal- 

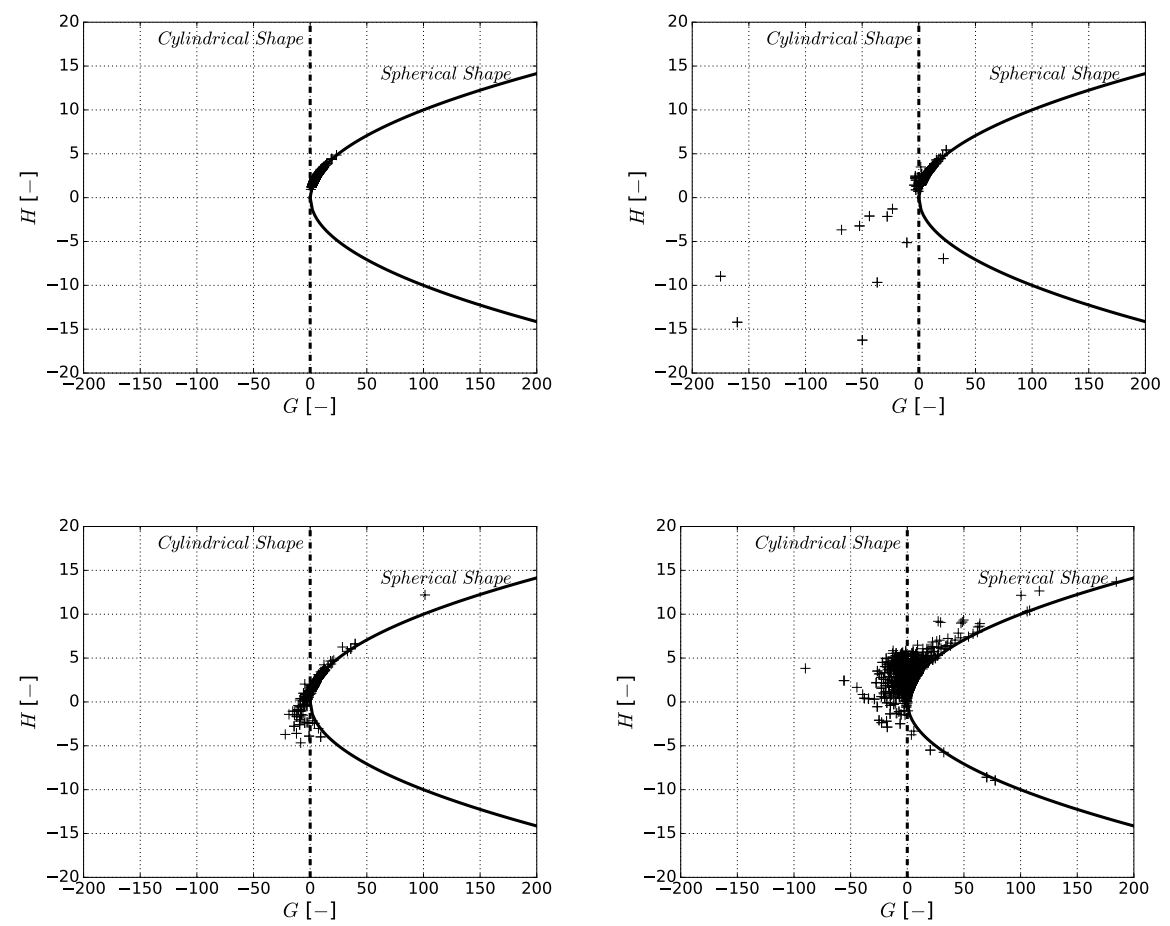

Figure 12: Mean curvature in function of Gauss curvature. Top left : at $t_{a}$; Top right : at $t_{b}$; Bottom left : at $t_{c}$; Bottom right : at $t_{d}$. Solid line represents $\kappa_{1}=\kappa_{2}$ (sphere) and the dashed line represents $G=0$ (cylinder).

culation the liquid-gas interface associated for each class of $H$. Such procedure has been gathered for nearly $15 \tau^{*}$ in order to reduce the statistical noise and to obtain a time-averaged distribution. Black dotted lines represent the mesh sizing, which can be considered as a numerical limit in curvature calculation. A similar evolution has been obtained also for the $\operatorname{PDF}(G)$, which is not shown here for the sake of clarity. The peak of $\operatorname{PDF}(H)$ is located at positive values since a dilute spray is here considered, whereas the probability to have gas bubbles (i.e. $H<0$ ) is negligible. Starting from such distribution, much more information about the evolution of the liquid-gas interface can be now extracted. For instance, the spray NDD can be now derived, associating to each curvature class $\left(H_{i}\right)$ the corresponding number of spherical droplets $\left(N_{d . i}\right)$ characterized 


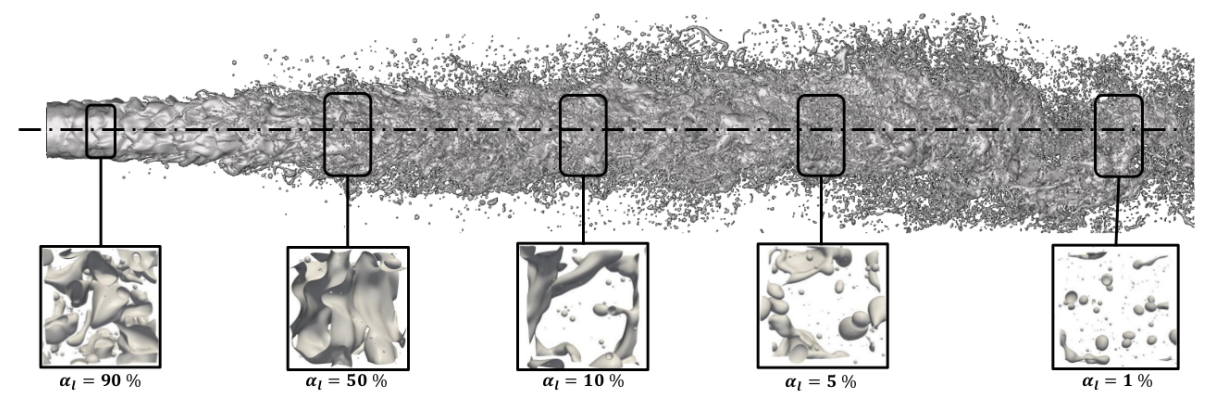

Figure 13: Regions considered for the extraction of curvature distribution all along the atomization process.
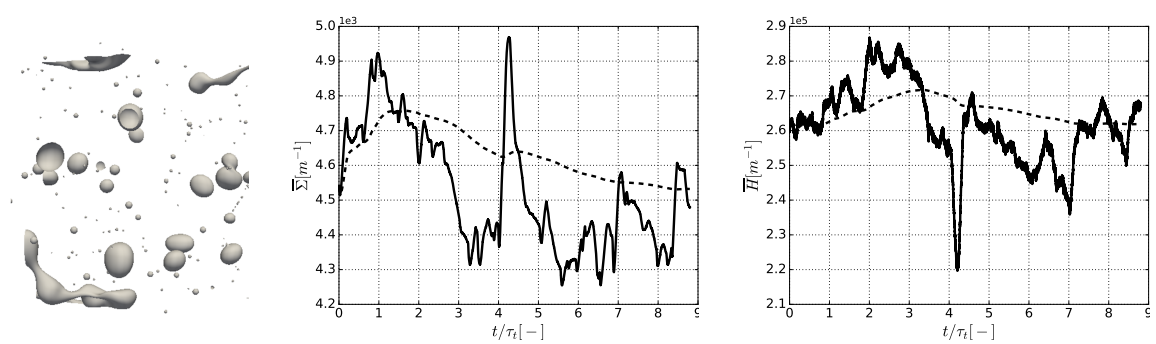

Figure 14: Visualization of liquid-gas interface for liquid volume fraction of 1\%, together with time evolution of $\bar{\Sigma}$ and $\bar{H}$. The solid lines represent the instantaneous signal of the mean value of $H$ and $\Sigma$. The dashed lines represent the time average of the mean value of $H$ and $\Sigma$.

by the class amount of surface $\left(A_{i}\right)$ collected during the calculation:

$$
N_{d, i}=\frac{A_{i}}{4 \pi\left(H_{i}\right)^{-2}}
$$

This represents clearly an abrupt approximation since each curvature class is associated to the corresponding spherical shape, but it highlights a direct link between curvature distributions and the NDD. Using this formulation, it has been possible to extract the NDD and the Volume based Drop size Distribution (VDD) shown in Figure 16 using solid black lines. The reader interested in the theoretical link between the NDD and the VDD is addressed to references $(\overline{\mathrm{Du}}-$ 380 mouchel, 2006; Babinsky and Sojka, 2002; Déchelette et al., 2011) where this 


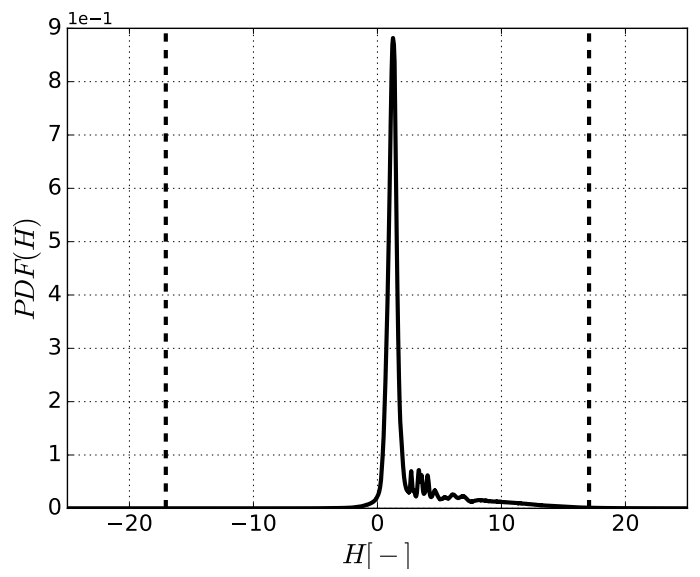

Figure 15: Time averaged PDF of mean curvature.

topic is discussed in detail. A double peaked NDD is obtained from numerical
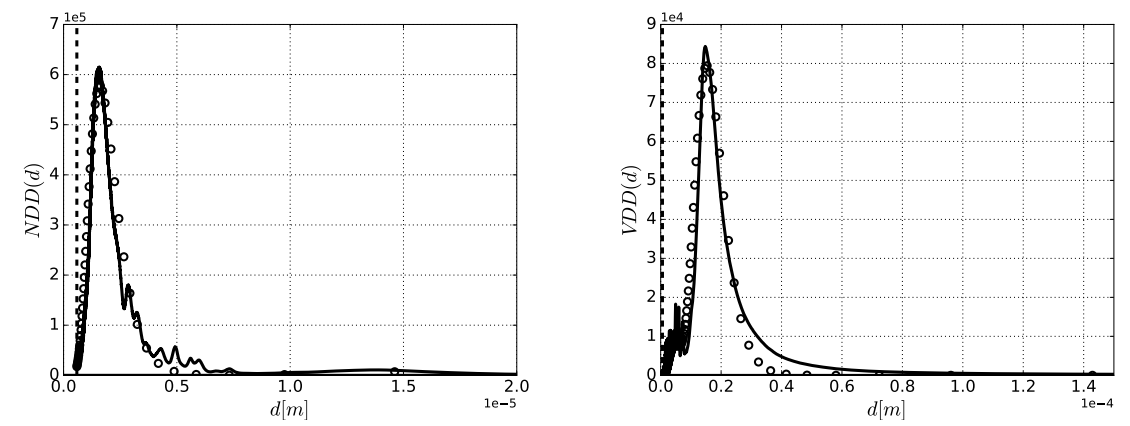

Figure 16: Extraction of NDD and VDD from $\operatorname{PDF}(H)$. Solid line : DNS data, dashed lines : $d x$ representation, $\circ$ : proposed log-normal distribution.

calculations. It is representative of the generation of a huge number of nearly spherical droplets together with few ligaments, which bring the major part of the liquid volume. Such small particles are generated through collisional events, which are enhanced by the turbulent liquid-gas interactions. The turbulence, acting on the liquid surface, increases the probability of collisions and determines the final spreading of the size distribution. 
Log-normal probability distributions are calculated to fit the DNS data : first two separate PDFs have been derived (i.e. $P D F_{1}$ for the diameter class 1e$08<\mathrm{d}[\mathrm{m}]<1 \mathrm{e}-05$ and $P D F_{2}$ for $\left.1 \mathrm{e}-05<\mathrm{d}[\mathrm{m}]<1.5 \mathrm{e}-04\right)$ and then the total probability distribution, calculated as $P D F_{\text {tot }}=V_{1} P D F_{1}+V_{2} P D F_{2}$ where $V_{1}$ and $V_{2}$ represent the liquid volume associated to each class, has been obtained (See Figure 16. It is worth noting that $V_{1}$ represents nearly the $5 \%$ of the total liquid volume, showing that, even for such low value of liquid volume fraction, the contribution of collisions is not at all negligible. The resulting log-normal $P D F_{\text {tot }}$, both in terms of NDD or VDD, reliably agrees with DNS data. Hence, starting from the $H$-PDF it has been possible to derive a NDD that is consistent with a standard drop-size distribution in the dilute regime like the log-normal one. Furthermore, it is worth pointing out that the mean value calculated for $P D F_{t o t}$ is coherent with the characteristic Sauter Mean Diameter (SMD) that can be calculated as $S M D=6 \alpha_{l} / \Sigma$. Such evidence enforces the idea that curvatures PDF can be reliably used to characterized the spray distribution both in terms of mean value and dispersion.

This comparison clearly shows that, in a test article nearly representative for 405 a dilute spray (i.e. $\alpha_{l}=1 \%$ ), a direct link between the spray NDD and the curvature evolution can be determined. However, beyond the characterization
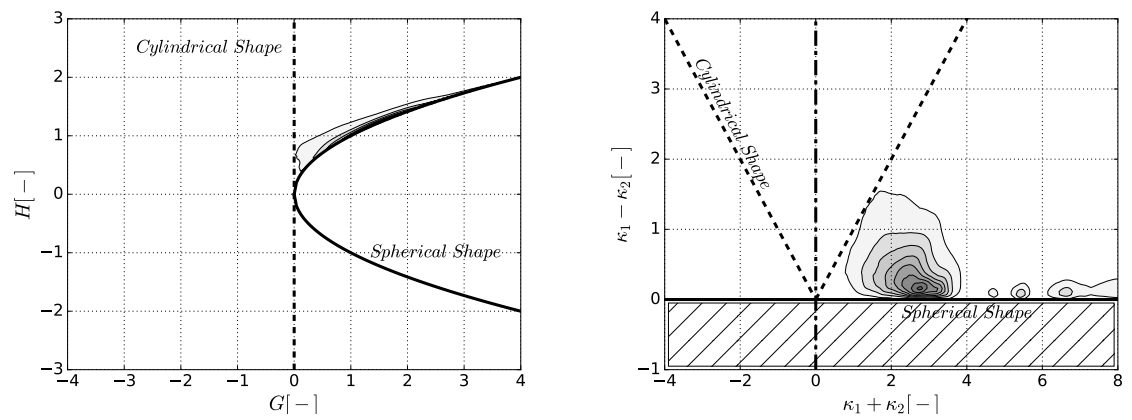

Figure 17: Joint $\operatorname{PDF}$ of the surface curvatures for $\alpha_{1}=1 \%$. Left : joint $\operatorname{PDF}(H, G)$, right: joint $\operatorname{PDF}\left(\kappa_{1}-\kappa_{2}, \kappa_{1}+\kappa_{2}\right)$. 
of the spray distribution, such DNS analysis can bring also further information about the curvature evolution that can be useful to better understand the topology of the liquid-gas interface under investigation and to give some hints about the curvature modeling. In particular, in Figure 17 the time averaged joint $\operatorname{PDF}(H, G)$ is shown together with the time averaged joint $\operatorname{PDF}$ of the $\left(\kappa_{1}-\kappa_{2}, \kappa_{1}+\kappa_{2}\right)$ space. On the joint $\operatorname{PDF}(H, G)$, the parabola represents the spherical shape (i.e. $H^{2}=G$ ) whereas the dashed line specifies a cylindrical topology (i.e. $G=0$ ). On the joint $\operatorname{PDF}\left(\kappa_{1}-\kappa_{2}, \kappa_{1}+\kappa_{2}\right)$, the dashed lines represent a cylindrical shape and the spherical shape is shown by the horizontal solid line. The vertical dash-dotted line separates bubbles topology from droplets topology. It is possible to extract several interesting information about the evolution of the liquid-gas interface looking at such joint PDF distributions of surface curvature:

- the $\operatorname{PDF}(H, G)$ shows that, in terms of time-averaged distribution, the topology of the liquid surface is mainly stretched along two directions, i.e. several points are collapsed on the parabola representative of the spherical shape and in a similar manner also the cylindrical asymptotic limit appears. Such bi-modal topological distribution derives from the presence of big cylindrical ligaments that, subjected on the action of the turbulence field, collide and generate a cloud of spherical droplets.

- the $\operatorname{PDF}\left(\kappa_{1}-\kappa_{2}, \kappa_{1}+\kappa_{2}\right)$ gives similar information in terms of timeaveraged properties with respect to the $\operatorname{PDF}(H, G)$, but, looking now at the contour iso-lines, it should be pointed out that the most probable value of the two principal curvatures is not associated to a spherical shape (i.e. it not collapsed on the $\kappa_{1}-\kappa_{2}=0$ line).

Hence, the joint PDFs, which can be extracted from surface curvatures, may give an idea about the topological evolution of the two phase flow together with some hints about the generated spray population. Considering these results, together with data already presented on the Rayleigh-Plateau test case, the capabilities of surface curvatures in describing the liquid breakup as well as its 
direct link with the NDD have been clarified.

Therefore, higher values of liquid volume fraction have been then investigated in order to understand how the curvatures evolve and if it is possible to determine 440 a general behavior of the curvatures PDFs all along the atomization process.

In Figure 18 the liquid-gas interface distribution together with the time averaged $\operatorname{PDF}(H)$ is shown for $\alpha_{l}=5 \%$. Clearly, much more ligaments are generated now due to the augmentation of the liquid volume inside the box with respect to the previous case: elongated and stretched cylindrical elements appear and from their turbulent breakup some spherical droplets are produced. The $\operatorname{PDF}(H)$ shows here an evolution in line with Figure 16 with a mode value shifted towards negative values of $H$. Now, the probability of finding $H<0$ is no more zero and this is linked to the presence of regions where gas bubbles are surrounded by the liquid phase leading to an opposite curvature sign.
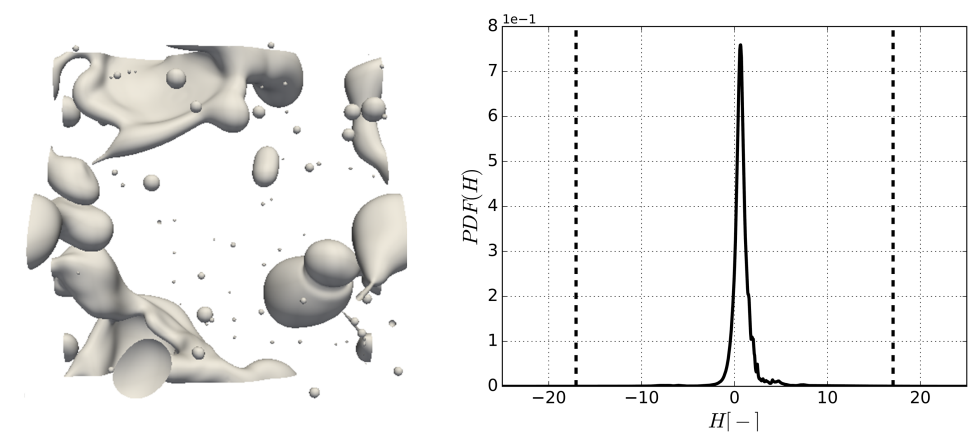

Figure 18: Left : Visualization of liquid-gas interface for liquid volume fraction of $5 \%$. Right : time averaged $\operatorname{PDF}(H)$.

In Figure 19 the joint $\operatorname{PDF}(H, G)$ and $\operatorname{PDF}\left(\kappa_{1}-\kappa_{2}, \kappa_{1}+\kappa_{2}\right)$ are reported for the $5 \%$ case. Considering the $\operatorname{PDF}(H, G)$, with respect to Figure 17 less points tends now to collapse along the parabola and the probability peak is strongly stretched along the line at $G=0$. This is again representative of the topological variation in the liquid shape from a sphere to a deformed and elongated cylinder while increasing the liquid volume fraction. In a similar fashion, looking at the $\operatorname{PDF}\left(\kappa_{1}-\kappa_{2}, \kappa_{1}+\kappa_{2}\right)$ the distribution is no more just collapsed on the spherical 

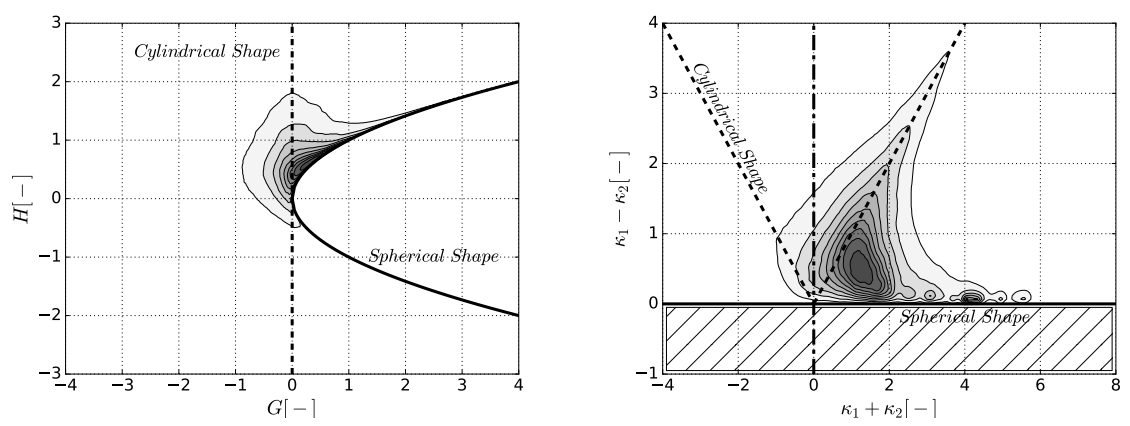

Figure 19: Joint $\mathrm{PDF}$ of the surface curvatures for $\alpha_{l}=5 \%$. Left : joint $\operatorname{PDF}(H, G)$, right : joint $\operatorname{PDF}\left(\kappa_{1}-\kappa_{2}, \kappa_{1}+\kappa_{2}\right)$.

topology, but tends to be straightened also along the line corresponding to a cylindrical shape $\left(\kappa_{2}=0\right)$, with the PDF peak moved away from the spherical asymptote with respect to the previous test point. This clearly shows that for $\alpha_{l}=5 \%$, the interface has a wider range of possible curvatures than the previous $\alpha_{l}=1 \%$ configuration, and is subject to more deformation and wrinkles.

Increasing further the liquid volume fraction, as shown in Figure 20 from the instantaneous snapshots from $\alpha_{l}=10 \%$ up to $90 \%$, an important number of continuous liquid structures appear and a set of bubbles is at the end generated for the very dense case. The time averaged $\operatorname{PDF}(H)$ as well as the joint
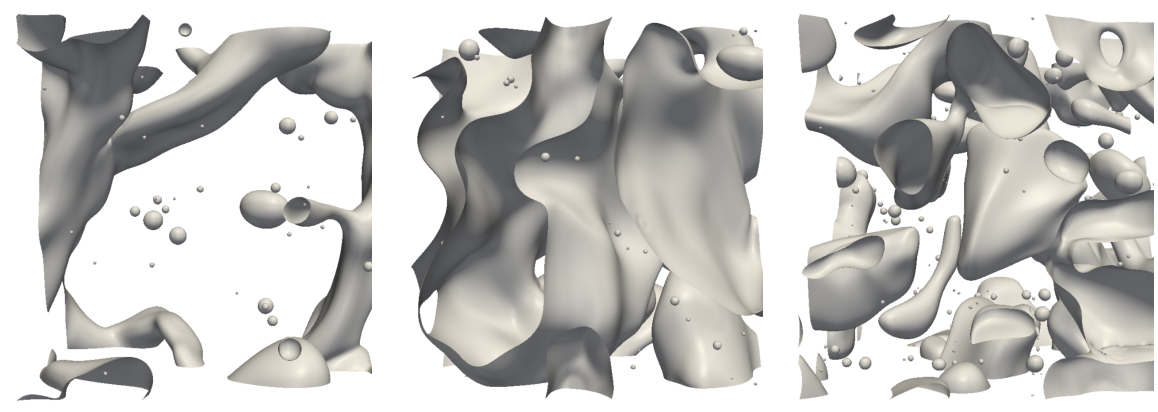

Figure 20: Visualization of liquid-gas interface for liquid volume fraction for $10 \%$ (left), $50 \%$ (center) and 90\% (right).

465

$\operatorname{PDF}(H, G)$ and $\operatorname{PDF}\left(\kappa_{1}-\kappa_{2}, \kappa_{1}+\kappa_{2}\right)$ are shown respectively in Figures 21 
and 22. Several considerations can be derived from these plots. For $\alpha_{l}=10 \%$, some pockets of gas are closed inside the continuous liquid structures and indeed, with respect to previous cases, now the probability of having $H<0$ is no more negligible.
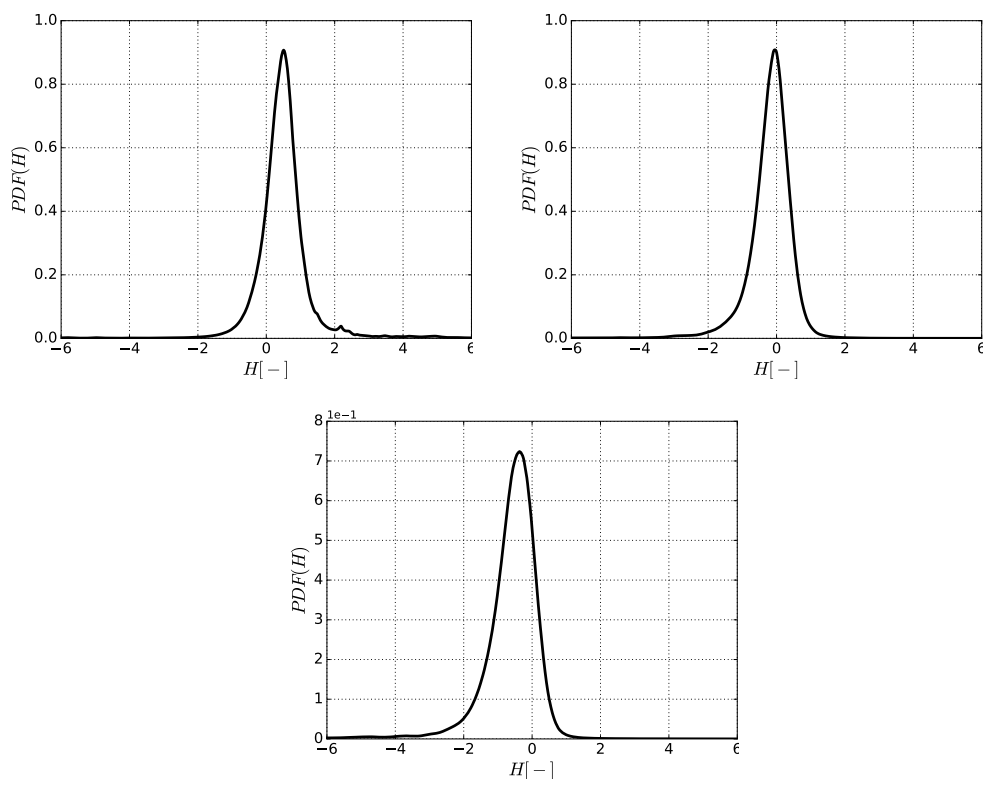

Figure 21: Time averaged PDF of mean curvature. Top left : $\alpha_{l}=10 \%$, Top right : $\alpha_{l}=50 \%$ , bottom : $\alpha_{l}=90 \%$.

The probability distribution of the mean curvature has a similar shape with respect to Figure 16 with the mode value that is shifted towards lower $\mathrm{H}$-values. The topology of the the interface is now strongly modified and the $\operatorname{PDF}(H, G)$ points out a leading cylindrical shape together with two clouds of points collapsed on the parabola. They represents the generation of a set of bubbles and droplets nearly spherical. In terms of principal curvatures, the peak is moved from the $\kappa_{1}=\kappa_{2}$ limit $\left(\kappa_{1}-\kappa_{2}=0\right)$, even if a significant stretch along the spherical line can be still pointed out.

As soon as the liquid volume fraction is further augmented, points characterized by a positive mean curvature disappear since the probability of having some iso- 

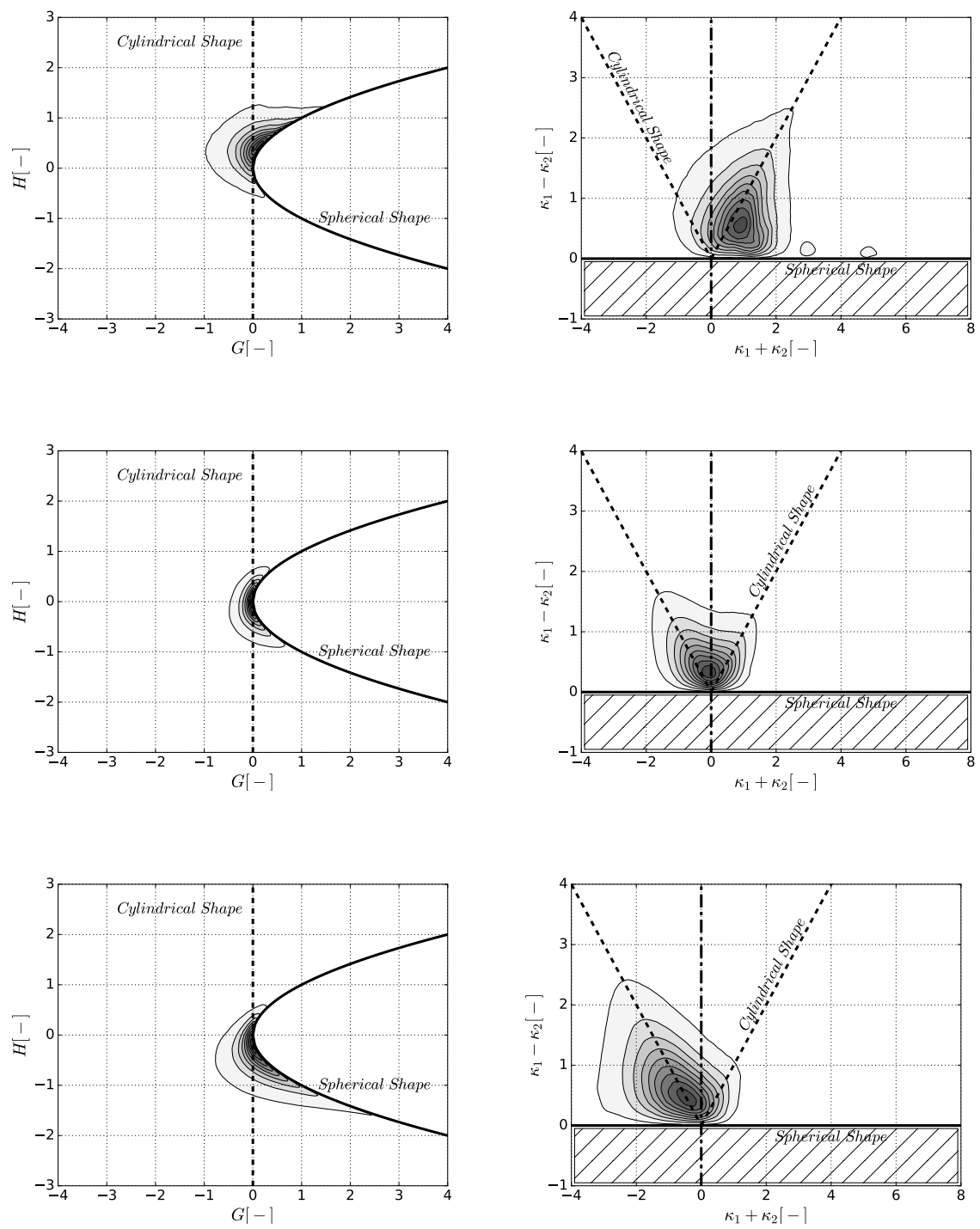

Figure 22: Time averaged joint $\operatorname{PDF}(H, G)$ (left) and $\operatorname{PDF}\left(\kappa_{1}-\kappa_{2}, \kappa_{1}+\kappa_{2}\right)$ (right) for $\alpha_{l}=10 \%$ (top), $\alpha_{l}=50 \%$ (center) and $\alpha_{l}=90 \%$ (bottom). 
lated droplets is now negligible. The imposed level of turbulent kinetic energy is not high enough to disrupt the ligaments and the liquid-gas interface preserves its continuity. The gas phase is trapped inside these structures and on the $(H, G)$ plot a huge amount of bubbles collapses on the spherical topology. Coherently, the principal curvatures change their sign. Finally, at $\alpha_{l}=90 \%$ the characteristic values of $H$ and $G$ are higher (i.e. bubbles with a smaller radius) and the $\operatorname{PDF}\left(\kappa_{1}-\kappa_{2}, \kappa_{1}+\kappa_{2}\right)$ changes completely its orientation and the system is stretched on the negative principal curvatures space. The HIT now behaves in terms of topology in a complementary manner with respect to the $10 \%$ case as shown also by the time averaged $\operatorname{PDF}(H)$, where the peak is moved towards negative values because of the generated smaller bubbles.

By comparing all the results shown so far, it is interesting to point out that the curvature evolution is completely consistent with the observed physical phenomena. Both the $\operatorname{PDF}(H)$ and the joint curvature probability distributions evolve all along the atomization process maintaining the same shape and moving the peak of the distribution from positive to negative values. Moreover, as far as the spray becomes dilute a link between the curvature evolution and a standard DSD is directly available. This surely represents a novel and general framework to study the atomization process and the obtained mathematical shape of the $\operatorname{PDF}(H)$ is particularly promising having in mind the introduction of such characteristics of the liquid-gas interface in the modeling context (for instance in the ELSA formalism).

\section{Conclusions}

This study shows how DNS of two-phase flows can be employed to improve understanding and modeling of primary atomization to be then used in RANS or LES framework. In particular, the study is aimed at enhancing the information available in ELSA framework through the introduction of liquid/gas interface curvatures. Firstly, on a mathematical point of view, an extended definition of the drop size distribution to be used all along the atomization pro- 
cess is proposed, leading to the so called surface curvature distribution. Hence, the extraction of surface curvatures is shown for simple configurations such a Rayleigh-Plateau instability and a droplet collision. Two different methods to extract the curvatures have been tested and validated. The evolution of Gauss and mean curvatures is analyzed in detail in order to show how the breakup process from a liquid jet instability to the final droplet can be easily described in terms of such surface geometrical properties. Then, the same procedure has been applied on the HIT, where several cases, varying the liquid volume fraction, have been studied. The analysis has been firstly focused on a dilute case (i.e. $\alpha_{1}=1 \%$ ) where the link between the DSD and SCD has been clarified. Indeed, starting from the $\operatorname{PDF}(H)$ it has been possible to extract a NDD and VDD that follow a standard log-normal distribution. By investigating higher values of the liquid volume fraction (up to 90\%), it has been shown that the SCD is defined even if droplets/bubbles or large liquid/gas structures are present. Joint PDFs of $H$ and $G$, as well as joint PDF of $\kappa_{1}-\kappa_{2}$ and $\kappa_{1}+\kappa_{2}$, have been investigated in this configuration and give a good insight on the behavior of the two-phase flows and the evolution of liquid (or gas) structures shapes. It has been found that curvature distributions are able to describe the transition from a dilute spray to a bubbly flow and in particular the $\operatorname{PDF}(H)$ shows a very similar shape in all the studied cases.

\section{Acknowledgements}

This work was granted access to the HPC resources of IDRIS, TGCC and CINES under the allocation A0032B06153 made by GENCI (Grand Equipement National de Calcul Intensif).

\section{References}

${ }_{535}$ Babinsky, E., Sojka, P. E., 2002. Modeling drop size distributions. Progress in energy and combustion science 28 (4), 303-329. 
Chesnel, J., Reveillon, J., Menard, T., Demoulin, F.-X., 2011. Large eddy simulation of liquid jet atomization. Atomization and Sprays 21 (9), 711-736.

Demoulin, F.-X., Reveillon, J., Duret, B., Bouali, Z., Desjonqueres, P., Menard, T., 2013. Toward using direct numerical simulation to improve primary breakup modeling. Atomization and Sprays 23 (11), 957-980.

Dumouchel, C., Dec. 2006. A New Formulation of the Maximum Entropy Formalism to Model Liquid Spray Drop-Size Distribution. Particle \&amp; Particle Systems Characterization 23 (6), 468-479.

Dumouchel, C., Aniszewski, W., Vu, T.-T., Ménard, T., Jun. 2017. Multi-scale analysis of simulated capillary instability. International Journal of Multiphase Flow 92, 181-192.

Dumouchel, C., Ménard, T., Aniszewski, W., Nov. 2015. Towards an interpretation of the scale diffusivity in liquid atomization process: An experimental approach. Physica A: Statistical Mechanics and its Applications 438, 612-624.

Duret, B., Luret, G., Reveillon, J., Menard, T., Berlemont, A., Demoulin, F.X., 2012. DNS analysis of turbulent mixing in two-phase flows. International Journal of Multiphase Flow 40 (0), 93 - 105.

Duret, B., Reveillon, J., Menard, T., Demoulin, F., 2013. Improving primary atomization modeling through DNS of two-phase flows. International Journal of Multiphase Flow Volume 55, 130-137.

Déchelette, A., Babinsky, E., Sojka, P. E., 2011. Drop Size Distributions. In: Handbook of Atomization and Sprays. Springer, Boston, MA, pp. 479-495, dOI: 10.1007/978-1-4419-7264-4_23.

560

Déjean, B., Berthoumieu, P., Gajan, P., Mar. 2016. Experimental study on the influence of liquid and air boundary conditions on a planar air-blasted liquid sheet, Part I: Liquid and air thicknesses. International Journal of Multiphase Flow 79, 202-213. 
Essadki, M., De Chaisemartin, S., Laurent, F., Massot, M., 2018a. High Order Moment Model for Polydisperse Evaporating Sprays Towards Interfacial Geometry. in revision for SIAM Journal on Applied Mathematics 1608.

Essadki, M., de Chaisemartin, S., Massot, M., Laurent, F., Larat, A., Jay, S., Sep. 2016. Adaptive Mesh Refinement and High Order Geometrical Moment Method for the Simulation of Polydisperse Evaporating Sprays. Oil \& Gas Science and Technology, Revue d'IFP Energies nouvelles 71 (5), 61.

Essadki, M., Drui, F., de Chaisemartin, S., Larat, A., Ménard, T., Massot, M., Oct. 2018b. Statistical modeling of the gas-liquid interface using geometrical variables: toward a unified description of the disperse and separated phase flows. in revision for International Journal of Multiphase Flow.

${ }_{575}$ Fox, R. O., 2012. Large-Eddy-Simulation Tools for Multiphase Flows. Annual Review of Fluid Mechanics 44 (1), 47-76.

Gorokhovski, M., Herrmann, M., 2008. Modeling Primary Atomization. Annual Review of Fluid Mechanics 40 (1), 343-366.

Kang, M., Fedkiw, R. P., Liu, X., 2000. Boundary conditions capturing method for multiphase incompressible flow. Journal of Scientific Computing 3, 323360 .

Kindlmann, G., Whitaker, R., Tasdizen, T., Moller, T., 2003. Curvature-based transfer functions for direct volume rendering: Methods and applications. In: Visualization, 2003. VIS 2003. IEEE. IEEE, pp. 513-520.

${ }_{585}$ Lalanne, B., Villegas, L. R., Tanguy, S., Risso, F., Nov. 2015. On the computation of viscous terms for incompressible two-phase flows with Level Set/Ghost Fluid Method. Journal of Computational Physics 301, 289-307.

Lebas, R., Menard, T., Beau, P. A., Berlemont, A., Demoulin, F. X., 2009. Numerical simulation of primary break-up and atomization: DNS and modelling study. International Journal of Multiphase Flow 35 (3), 247 - 260. 
Liu, X.-D., Fedkiw, R. P., Kang, M., 2000. A Boundary Condition Capturing Method for Poisson's Equation on Irregular Domains. Journal of Computational Physics 160 (1), $151-178$.

Luret, G., Menard, T., Blokkeel, G., Berlemont, A., Réveillon, J., Demoulin, F., 2010. Modeling collision outcome in moderately dense spray. Journal of Atomization and Spray.

Meyer, M., Desbrun, M., Schröder, P., Barr, A. H., others, 2002. Discrete differential-geometry operators for triangulated 2-manifolds. Visualization and mathematics $3(2), 52-58$.

Ménard, T., 2007. Développement d'une méthode Level Set pour le suivi d'interface, application de la rupture de jet liquide. Ph.D. thesis, Université de Rouen.

Ménard, T., Tanguy, S., Berlemont, A., May 2007. Coupling level set/VOF/ghost fluid methods: Validation and application to 3d simulation

605 of the primary break-up of a liquid jet. International Journal of Multiphase Flow 33 (5), 510-524.

Navarro-Martinez, S., Jul. 2014. Large eddy simulation of spray atomization with a probability density function method. International Journal of Multiphase Flow 63, 11-22.

Sussman, M., Smith, K. M., Hussaini, M. Y., Ohta, M., Zhi-Wei, R., Feb. 2007. A sharp interface method for incompressible two-phase flows. Journal of Computational Physics 221 (2), 469-505.

Tanguy, S., Berlemont, A., Sep. 2005. Application of a level set method for simulation of droplet collisions. International Journal of Multiphase Flow 31 (9), $1015-1035$.

Vallet, A., Borghi, R., 1999. Modélisation eulerienne de l'atomisation d'un jet liquide. Comptes Rendus de l'Académie des Sciences-Series IIB-MechanicsPhysics-Astronomy 327 (10), 1015-1020. 
Vallet, A., Burluka, A. A., Borghi, R., 2001. Development of a Eulerian model for the "Atomization" of a liquid jet. Atomization and Sprays 11 (6), 619-642.

Vaudor, G., Ménard, T., Aniszewski, W., Doring, M., Berlemont, A., Jul. 2017. A consistent mass and momentum flux computation method for two phase flows. Application to atomization process. Computers \& Fluids 152, 204-216.

Warncke, K., Gepperth, S., Sauer, B., Sadiki, A., Janicka, J., Koch, R., Bauer, 625 H. J., May 2017. Experimental and numerical investigation of the primary breakup of an airblasted liquid sheet. International Journal of Multiphase Flow 91, 208-224.

Williams, F. A., 1958. Spray Combustion and Atomization. Physics of Fluids $1(6), 541$. 